\title{
Modeling Interaction Effects in Polarization: Individual Media Influence and the Impact of Town Meetings
}

\author{
Eric Pulick ${ }^{1}$, Patrick Korth ${ }^{2}$, Patrick Grim ${ }^{3}$, Jiin Jung ${ }^{4}$ \\ ${ }^{1}$ Department of Mechanical Engineering, G. G. Brown Laboratory, 2350 Hayward, Ann Arbor, MI, 48109, United \\ States of America \\ ${ }^{2}$ Department of Electrical Engineering and Computer Science, 1301 Beal Ave., Ann Arbor, MI, 48109, United \\ States of America \\ ${ }^{3}$ Center for Study of Complex Systems, University of Michigan, 321A West Hall, 1085 Ann Arbor, MI, 48109-1107, \\ United States of America \\ ${ }^{4}$ Department of Psychology, Claremont Graduate University, 123 East Eight Street, Claremont, CA, 91711, \\ United States of America \\ Correspondence should be addressed to patrick.grim@stonybrook.edu \\ Journal of Artificial Societies and Social Simulation 19(2) 1, 2016 \\ Doi: 10.18564/jasss.3021 Url: http://jasss.soc.surrey.ac.uk/19/2/1.html \\ Received: 25-05-2015 Accepted: 25-01-2016 Published: 31-03-2016
}

\begin{abstract}
We are increasingly exposed to polarized media sources, with clear evidence that individuals choose those sources closest to their existing views. We also have a tradition of open face-to-face group discussion in town meetings, for example. There are a range of current proposals to revive the role of group meetings in democratic decision-making. Here, we build a simulation that instantiates aspects of reinforcement theory in a model of competing social influences. What can we expect in the interaction of polarized media with group interaction along the lines of town meetings? Some surprises are evident from a computational model that includes both. Deliberative group discussion can be expected to produce opinion convergence. That convergence may not, however, be a cure for extreme views polarized at opposite ends of the opinion spectrum. In a large class of cases, we show that adding the influence of group meetings in an environment of self-selected media produces not a moderate central consensus but opinion convergence at one of the extremes defined by polarized media.
\end{abstract}

Keywords: Polarization, Media, Opinion, Social Networks, Town Meetings, Reinforcement

\section{Introduction}

1.1 Both political media and the character of public meetings have changed radically over the past half-century. The media of the 1960 s were dominated by the largely interchangeable news coverage of CBS, ABC, and NBC; today the opposing positions of Fox and MSNBC are a major media presence, as are the myriad internet sites individuals can and do self-select in order to reinforce a given position (lyengar \& Hahn 2009; Sunstein 2009; Stroud 2011; Pew Research Center 2012; Prior 2013). At the same time, there has been a dramatic decline in the role of fraternal and other groups that afforded face to face discussion among local groups in ways that often cross-cut economic and political differences - the Rotary Club, Elks, Lions, American Legion, Masons, and Order of the Eastern Star (Skocpol 2013; Bishop 2008). There have been a range of contemporary appeals for increased group deliberations in the form of citizen forums, 'public spheres,' or 'mini-publics' (Habermas 1996; Fishkin 1991, 1995, 2009; Bryan 2004; Fung 2006; Warren \& Pearse 2008; Warren 2009; Niemeyer 2011; Grönlund et al. 2013).

1.2 Prospects for individual self-selection of belief-tailored media have increased. Prospects for deliberative group discussion in the tradition of town meetings have seemed to decrease, with arguments that it should be revived. What consequences can we expect for political polarization from changes in these two variables? What effects can we expect self-selected media sources have on opinion polarization in a population? What effects can we 
expect town meetings to have? The focus of our study here: in what ways might we expect these two opinionshaping forces to work together or in opposition, either moderating or amplifying opinion polarization across a population?

1.3 We construct an agent-based model in the search for some insight into dynamics that may be operational in our contemporary political environment. The basic mechanisms of that model are patterned on established psychological principles. Its core is in the tradition of Axelrod's model of cultural assimilation and dissociation, directly targeting polarization in terms of the basic psychological principles of homophily and imitation (Axelrod 1997; Castellano et al. 2000; Klemm et al. 2003a,b; Flache \& Macy 2006). By homophily, people are more likely to interact with those who share many of their cultural attributes (McPherson et al. 2001). By imitation, interactions between people tend to increase the number of attributes they share (Festinger 1954).

1.4 To those mechanisms we add self-selected media and the force of group consensus as social reinforcers. Scheduled reinforcement is a stalwart of behavioral psychology (Ferster \& Skinner 1957; Zeiler 1977; Morgan 2010). The effectiveness of a reinforcer in strengthening or weakening a particular behavior depends not merely on how many times it has been given but on the schedule of reinforcement. The effectiveness with which a target behavior is shaped or eliminated can depend not only on whether reinforcers are negative or positive but on patterns of multiple combinatory reinforcers given before and/or after the target behavior (Madden 2013; Morgan 2010; Zeiler 1977). Skinner was clear that reinforcers may well be cultural, evolved in a social environment in precisely the sense employed here: "Human behavior is the joint product of (i) the contingencies of survival responsible for the natural selection of the species and (ii) the contingencies of reinforcement responsible for the repertories acquired by its members, including (iii) the special contingencies maintained by an evolved social environment, culture" (1981, p. 502).

1.5 It is undoubtedly true both that culture is the product of individual decisions and that individual decisions are often the product of cultural forces. The social dynamics may well be complex (Mason et al. 2007). In this paper we focus on mechanisms of scheduled and multiple social reinforcement in order to track the impact on polarization of interaction effects between self-selected media and the influence of group consensus within town meetings, 'public spheres,' or 'mini-publics' (Habermas 1996; Fishkin 1991, 1995, 2009; Fung 2006; Warren \& Pearse 2008; Warren 2009; Niemeyer 2011; Grönlund et al. 2013).

1.6 It is well known that the effect of superimposed schedules of reinforcement can differ significantly from the effect of individual schedules alone and from any simple linear addition of effects (Wilkie 1972; Brechner 1977; Brechner \& Linder 1981; Williams 1983, 1994). But to our knowledge the interaction effects of superimposed reinforcement schedules have not been broached in social modeling. Our results indicate complex interaction effects between self-selected media and town meetings that would not have been expected from considering either alone.

\section{The Model}

2.1 The model we use is a variation and extension of Axelrod (1997) that also incorporates selected features from Deffuant et al. (2000, 2002, 2004). We offer a condensed characterization followed by explanation of the motivation for particular features and ties to background literature. Model code is available here.

2.2 Agents on a lattice each have a set of political beliefs, modelled as a list of 5 real-valued numbers from 1 to 10. Three types of interactions occur which may shift an agent's political beliefs: neighbor interactions, media interactions, and the group interactions we term 'town meetings.' Interaction of each type occurs with a probability calculated from the over-all similarity between an agent and the target (neighbor, media source, or average view of agents in town meeting). Similarity is found by summing the difference of each political belief, normalizing by dividing by the maximum possible difference, then subtracting from 1 . Using integer values for a simple example, given that belief values range from 1 to 10 , the belief sets [13543] and [55555] have a similarity $1-[(5-1+5-3+5-5+5-4+5-3) /((10-1) * 5)]=0.80$. The sets $[12121]$ and $[55555]$ are less similar, and have a similarity of $1-(5-1+5-2+5-1+5-2+5-1) /((10-1) * 5)=0.60$. If the similarity between an agent and the target is $X$, the agent has an $X \%$ chance of shifting one of its political beliefs. We randomly choose a single political belief that differs between the agent and the target, shifting that belief of the agent halfway to that of the target. For example, if the agent's belief is 2.07 and the other belief is 6.89 , the agent's political belief value is set to $2.07+(6.897-2.07) * 0.5=4.48$. For computational simplicity, values are rounded to the nearest hundredth.

2.3 The first form of interaction we investigate is between neighbors. At each step or 'tick' of the simulation, each agent chooses a random one of its 8 neighbors. Given a similarity of $X$ to that neighbor, as outlined above, the 
A Typical Initial Set of Cultures

\begin{tabular}{llllllllll}
\hline \hline 74741 & 87254 & $\frac{82330}{17993}$ & 22978 & 82762 & 87476 & 26757 & 99313 & 32009 \\
01948 & 09234 & 67730 & 89130 & 34210 & 85403 & 69411 & 81677 & 06789 & 24042 \\
49447 & 46012 & 42628 & 86636 & 27405 & 39747 & 97450 & 71833 & 07192 & 87426 \\
22781 & 85541 & 51585 & 84468 & 18122 & 60094 & 71819 & 51912 & 32095 & 11318 \\
09581 & 89800 & 72031 & 19856 & 08071 & 97744 & 42533 & 33723 & 24659 & 03847 \\
56352 & 34490 & 48416 & 55455 & 88600 & 78295 & 69896 & 96775 & 86714 & 02932 \\
46238 & 38032 & 34235 & 45602 & 39891 & 84866 & 38456 & 78008 & 27136 & 50153 \\
88136 & 21593 & 77404 & 17043 & 39238 & 81454 & 29464 & 74576 & 41924 & 43987 \\
35682 & 19232 & 80173 & 81447 & 22884 & 58260 & 53436 & 13623 & 05729 & 43378 \\
57816 & 55285 & 66329 & 30462 & 36729 & 13341 & 43986 & 45578 & 64585 & 47330 \\
\hline
\end{tabular}

NOTE: The underlined site and the site to its south share traits for two of the five cultural features, making a cultural similarity of $40 \%$.

Figure 1: An Axelrod lattice of cultures, consisting of 9 traits within 5 features Axelrod (1997).

agent has an $X \%$ probability of changing one of its political beliefs to match a different belief of its neighbor '. The second form of interaction is between agents and polarized media. Here we use two constant agents at opposite extremes of the political spectrum, one with all values at 1.00 , another with all values at 10.00 . Given a similarity $X$ to a media source, agents interact with probability $X$ with that media source that is over-all most similar, updating a randomly chosen belief as in the case of neighbor interaction. The third form of interaction is in town meetings, simulated by choosing a random position in the array and a distance radius of cells. Agents within that radius are identified as meeting participants. The political beliefs of the participants are averaged together and each participant then adjusts each of its values halfway toward the values of that group prototype.

2.4 Baseline simulations use 'word of mouth' interactions with neighbors. At sampling intervals we record the distribution of agents' averaged belief values, allowing us to use standard deviation in order to track the pattern of belief distribution over time. Against a background of neighbor interactions at each tick of the simulation, we then independently record the changes in belief distributions produced by (i) polarized media interaction of a fixed $10 \%$ of the population at different time intervals, and (ii) 'town meetings' of different radii at different time intervals. Because our main interest is in the interaction of media and town meetings, we report different interval settings for the two that show clear interactive effects on polarization.

2.5 Our final analysis attempts to gauge the scope of these effects. In our initial simulations it difficult to separate scheduling effects-how often town meetings occur, for example - from population proportion effects - what proportion of the population is involved in town meetings. In our final analysis we eliminate the scheduling effect by enforcing the selected interaction mechanisms each tick, varying only the proportion of the population affected. With neighbor interaction as a constant background, the proportion of the population interacting with media sources can vary from 0 to $100 \%$ as the proportion of the population interacting in town-meeting style varies from 0 to $100 \%$ as well. Using standard deviation together with the global average of agents' beliefs we are able to graph different polarization patterns across combinations of percentage parameters, confirming in greater definition the scope of the interaction effects identified in the earlier simulations.

\section{Opinion Updating: Adapting the Axelrod Model}

2.6 In Axelrod's model of cultural diffusion, agents in a bordered lattice (initially $10 \times 10$ ) are characterized by a representation of 'traits' on each of a small number of 'features' (see Figure 1). Axelrod thinks of traits, represented as digits 0 through 9 , as alternative cultural choices within, say, five cultural categories: alternative choices of color for particular items of clothing, for example. A series 87305 is intended to represent a 'culture' of different trait choices on a given set of features. We incorporate the multiple features of the Axelrod model as a major feature of our own, though reinterpreted in terms of suites of political opinions rather than aspects of a culture.

2.7 Axelrod attempts to capture two simple psychological principles of social influence: that people interact more with those who are like them, and that people come to hold views more like the views of those with whom they interact (Festinger 1954; McPherson et al. 2001). His core mechanism is one in which agents interact with bordering neighbors in the array with a probability proportionate to the number of features on which they share identical traits. On each round, a random agent and one of its neighbors are chosen for possible interaction. A pair with codes 87305 and 87392 will have a $60 \%$ chance of interaction; neighbors with codes 87305 and 89246 will have only a $20 \%$ chance. Should interaction take place, the original agent changes one of its mismatched 
traits (randomly chosen) to match that of its neighbor. If 87305 is the original agent and 87342 is its chosen neighbor, for example, the original agent may change its code to 87345 .

2.8 Using homophily and imitation as basic principles of social influence, we follow Axelrod in using sets of multiple values. We reinterpret these as modeling sets of political views rather than cultural features, with belief dynamics modeled as change across the multiple elements of those belief suites. In this respect the model contrasts with models written in terms of single values, whether discrete or continuous, characteristic of virtually all other traditions in the literature (Galam 1997; Kacperski \& Hotyst 2000; Hegselmann et al. 2002; Deffuant et al. $2000,2002,2004)$. In contrast with Axelrod, we model those multiple values in line with the intuitions regarding graduated opinion that guide the Deffuant tradition (Deffuant et al. 2000, 2002, 2004; McKeown \& Sheehy 2006). Features are treated as opinion topics rather than aspects of a culture, with trait values conceived as a spectrum of real values from 1 to 10 representing alternative positions on those topics.

2.9 We adapt rules for interaction and updating accordingly. In Axelrod's model, features are taken as aspects of a culture and distinct traits function simply as distinct alternatives. An agent's trait value on feature 5 is either distinct from that of its neighbor or not: a trait value of 8 is no closer to an alternative of 9 than is a trait value of 2. In our more sociopolitical interpretation, a series 8.027.563.190.615.97 will represent a particular configuration of shaded positions on, say, gun control, abortion, foreign policy, gay marriage, and immigration, ranging perhaps from conservative to liberal. In our modeling, a position of 3.07 on gun control is closer to 2.67 than it is to 9.56 (see also Flache \& Macy 2006).

2.10 Agents interact with a probability that matches their similarity, but that similarity is measured not in terms of full trait matches on features, as in Axelrod, but on trait proximity across the set of features as a whole. As outlined above, we sum the total differences in trait values across the entire set of features, dividing that by the maximum possible difference. In Axelrod's model, the probability of interaction between individuals will be zero when they match on no features-an aspect crucial to his modeling of polarization. In our model, because dissimilarity is measured by belief distance rather than trait match, probability of interaction virtually never reaches absolute zero. When agents do interact, moreover, the alteration of a randomly chosen point of disagreement will not be a sudden jump to the opinion value of the target. Our updating uses a slide of $50 \%$ instead: on the randomly chosen position, the agent's opinion will move halfway toward that of the target. Unlike bounded confidence models (Dittmer 2001; Hegselmann et al. 2002; McKeown \& Sheehy 2006), but in line with relative agreement variations (Deffuant et al. 2004), we think it most realistic to avoid any sharp threshold for influence: any degree of similarity across belief suites allows for some probability of interaction and graduated belief change.

2.11 We follow both the Axelrod and Deffuant traditions in modeling agent interaction on a lattice, with the influence of immediate neighbors as a component. In modeling the influence of deliberative group discussion, however, we also expand areas of influence beyond immediate neighbors to larger 'town meeting' patches in the lattice. With the precedent of (McKeown \& Sheehy 2006) we also introduce polarized media influence, but with a mechanism intended to model self-selected exposure. Our focus over all is on the combined influence of these forces when taken together: interaction effects of self-selected media together with town meetings as social reinforcers.

2.12 The configuration of the model, incorporating these features, is shown in Figure 2. Agents start with randomized traits, interacting with probabilities geared to over-all similarity and updating as a half-way approximation on a randomly chosen feature. Background histograms using intervals of 0.25 show the basic shape of aggregate values on features individually and combined, with color coding for liberal (red) or conservative (blue) leanings of agents averaged over all features.

\section{Schedules of Social Reinforcement: Town Meetings and Self-selected Media}

2.13 We expand the model in our more explicitly political interpretation to explore two forms of social influence, acting either independently or together. We model self-chosen media influence as interaction with unchanging external opinion configurations chosen on the basis of similarity to an agent's beliefs. We model town meetings as influence within larger clusters of neighbors. How does each of these forces shape public opinion? Most importantly, we want to explore what kind of interaction effects appear when both media influences and town meetings are in play simultaneously.

2.14 Models intended to include the effect of mass media have used both Axelrod's and Deffuant's bounded confidence models as a base (Shibanai et al. 2001; González-Avella et al. 2007), with some attention to both extremity of media influences and scheduling or 'broadcast ratio' (McKeown \& Sheehy 2006). What has not been well 

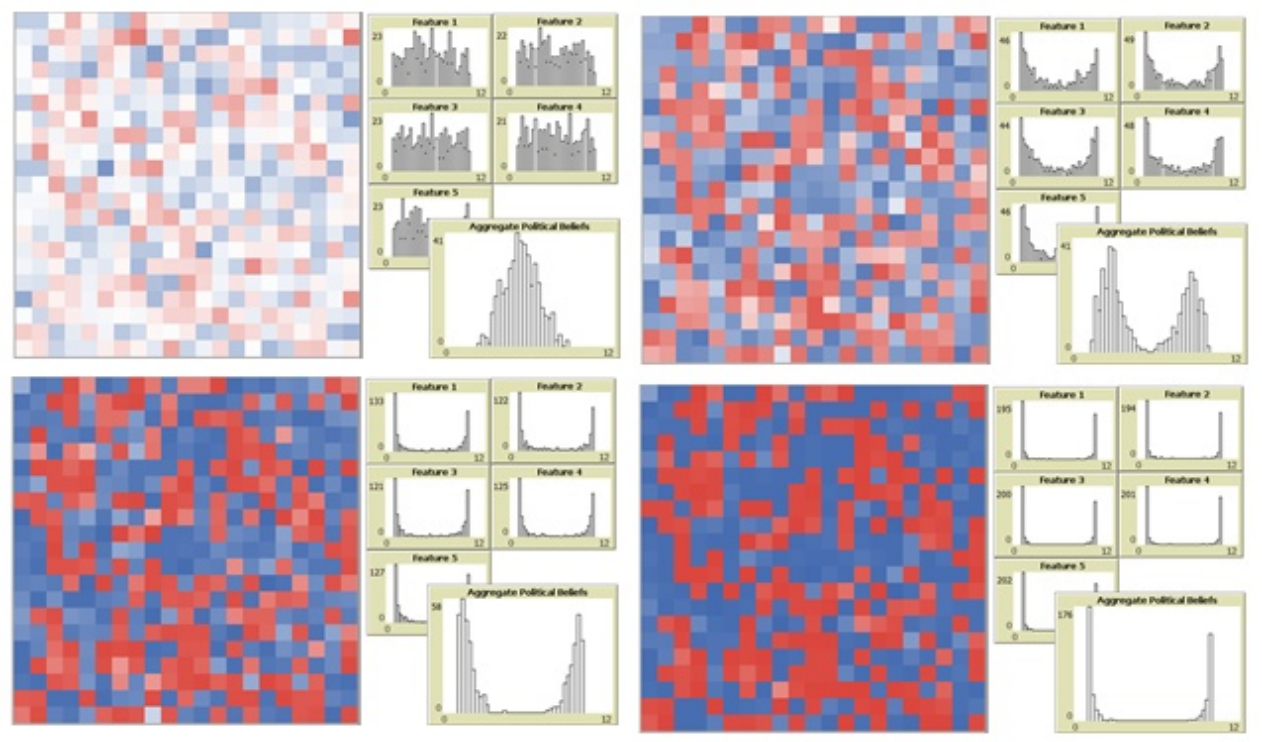

Figure 2: An Axelrod lattice of cultures, consisting of 9 traits within 5 features (Axelrod 1997). A form of the current model in which traits are treated as continuous values, with probabilities of interaction in terms of over-all similarity and updating as half-way approximation on a random feature. In this example $10 \%$ of the population is also exposed to a closest media source, as outlined below. Generations 0, 30, 60 and 90 shown.

modeled is the well-recognized sociological effect of self-selected media (Sustein 2002; Sunstein 2009; Bennett \& Iyengar 2008; lyengar \& Hahn 2009; Stroud 2011; Levendusky 2013, 2014; Leeper 2014).

2.15 A clear feature of the contemporary broadcast and cable environment is the existence of politically polarized media sources: Fox News and MSNBC are prime players, showing a clear conservative and liberal slant respectively in their news reporting, their editorializing, and the combination of the two. It is to be expected that individuals will seek out those media sources that echo and support their own political leanings (lyengar \& Hahn 2009; Sunstein 2009; Stroud 2011; Prior 2013; Pew Research Center 2012). The predictable result will be a strengthening of the initial leaning and reinforcement of those views that correspond to the slant of the media source chosen. Given a small number of media sources that are themselves polarized, self-selection of media can be expected to have a polarizing effect on public opinion at large ${ }^{2}$.

2.16 We follow Mckeown \& Sheehy (2006) in modeling two polarized media sources, though with continuous influence across elements in a suite of beliefs rather than a threshold cut-off of influence in a single belief ${ }^{3}$. One media source has opinions at 1.00 on the scale of all topics; the other has opinions at 10.00 for all topics. Like McKeown \& Sheehy (2006) we explored variations in degrees of polarization between media sources, but found the simple fact that media sources are symmetrically polarized is what proves crucial, rather than the specific points of polarization. Because results are similar at different separations we report just the extreme case here.

2.17 Individuals 'self-select' that media source that is most similar to their belief set over all, interacting with that media source with a probability based on over-all similarity. The fact that individuals most similar in opinion to a media source will have the highest probability of interaction and thus influence by that source accords perfectly with recent results on the effect of polarized media. Both Fiorina \& Levendusky (2006); Levendusky $(2013,2014)$ and Leeper $(2014)$ offer experimental evidence that it is individuals with opinions at extremes that are those most influenced by extreme media.

2.18 Previous work has not included the effect of citizen forums, 'public spheres,' or 'mini-publics' in the tradition of town meetings, much touted in aspects of political science (Habermas 1996; Fishkin 1991, 1995, 2009; Bryan 2004; Fung 2006; Warren \& Pearse 2008; Warren 2009; Niemeyer 2011; Grönlund et al. 2013). The psychological processes of social influence and attitude change within a group have been well documented (Turner 1985). Typically, a group is cognitively represented in terms of a group prototype, a set of attributes taken not only to describe the group but to prescribe how people should feel, think, and behave as group members, and how they should evaluate themselves and others. Social influence occurs as people assimilate themselves to the prototype of the group they belong to. Drawing on this psychological principle, we have each agent in a modeled group or town meeting interact with a prototypical position constructed by averaging values on each opinion topic across the whole, shifting a value to a point halfway between theirs and the prototypical position on each feature. 
2.19 Word of mouth influence is restricted to each cell's immediate neighbors. We model group influence in the town meeting tradition using larger areas of the array in which each cell is influenced by a prototypical position that consists of values on each opinion topic that are simply the average for all members of the group. As a participant in a group meeting, a cell updates values for each of its opinion topics by shifting to a point halfway between its original position on that topic and the group average represented in the prototype. We also explore how the size of group meetings may affect public opinion formation. Within an array of 400 cells we treat a small group as one in which individuals within a radius of 4 of that point interact, giving a group of 49 individuals. A medium group meeting is set at a radius of 7 , with 149 individuals. A large citizen forum takes a radius of 10, incorporating 317 individuals. With larger arrays using groups of the same population percentage we have found comparable results.

2.20 What we consider most significant in the model offered here are prospects for studying the interaction of selfselected media and group influence in social communication and opinion change. It is clear from the psychological literature that superimposing schedules of reinforcement from different stimuli can produce results that are importantly different from the individual stimuli schedules by themselves and from any linear addition of their effects (Ferster \& Skinner 1957; Wilkie 1972; Brechner 1977; Brechner \& Linder 1981; Williams 1983, 1994). Here we broach the interaction effects of superimposed reinforcement schedules in the context of social modeling. What the results show are complex interaction effects between self-selected media and group or town meetings that would not have been expected from considering either alone.

\section{Results}

3.1 We first examine how each of three social reinforcers (word of mouth, self-selected media, and town meetings) shapes public opinions when operating individually. We next explore interaction effects of the social reinforcers by applying different reinforcement schedules. We conclude by testing the robustness of observed effects across a full range of parameter combinations.

\section{Word of Mouth}

3.2 Agents across a $20 \times 20$ array start with randomized traits 1 through 10 in each of 5 feature slots ${ }^{4}$. A random agent and one of his neighbors are chosen each generation, with a probability of interaction given by their trait proximity across all features, as outlined above. If the two do interact, one randomly chosen trait of the agent is moved by a slider of $50 \%$ toward that of its neighbor.

3.3 Where agents update merely by word of mouth communication, interacting with their more similar neighbors and becoming more similar as a result, opinion across the population as a whole converges to an increasingly tight and increasingly uniform central position on the opinion scale.

3.4 Figure 3 shows the evolution of a typical random array. Background histograms indicate convergence at a central value for each of 5 features. The aggregate histogram and color coding show that convergence in terms added values for each agent across all 5 features.

3.5 Standard deviation can be used as a simple measure of histogram configuration. Simple convergence at the middle, as here, is indicated by the move to low standard deviation shown in Figure 4.

3.6 In our model, as in Axelrod's and other predecessors, one can often see the development of local color regions, light blue for one side of the spectrum and pink for the other, for example. In our model, however, because dissimilarity is measured by trait distance rather than trait match and without any sharp threshold at which influence is cut off, probability of interaction virtually never reaches absolute zero. Interaction therefore continues, as does the growth of consensus and similarity ${ }^{5}$.

\section{Town Meetings}

3.7 We retain word of mouth as a background social mechanism, adding group or town meetings as outlined above at a schedule of specific intervals: for every 25 ticks at which individuals interact by word of mouth with immediate neighbors, for example, a town meeting will occur in the radius of a randomly chosen point in the array. At a radius of 4, a small group includes 49 individuals of a 400 cell array. At a radius of 7 , a medium group consists of 149. A large group, at radius 10, incorporates 317 . When group influence of this form is added, of any radius, the result is still convergence to the center (Figure 5). Changes in other parameters result only in a difference in 

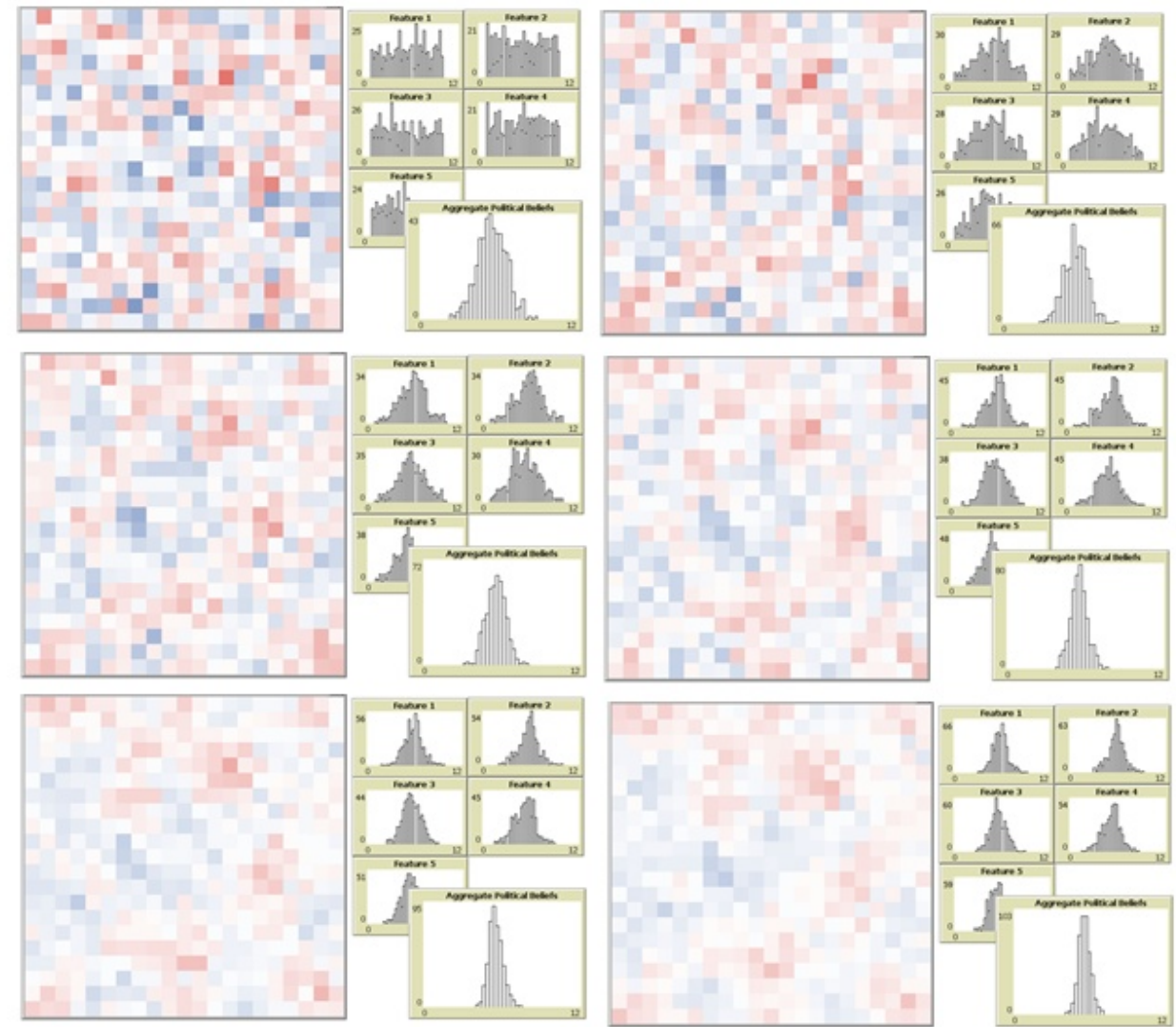

Figure 3: Increasing convergence on each feature with only word of mouth contact with neighbors. One pair chosen for interaction with probability in terms of over-all similarity each generation, images shown at intervals of $0,3000,6000,9000,12000$ and 15000 .

Standard deviation as a measure of convergence: Word of mouth

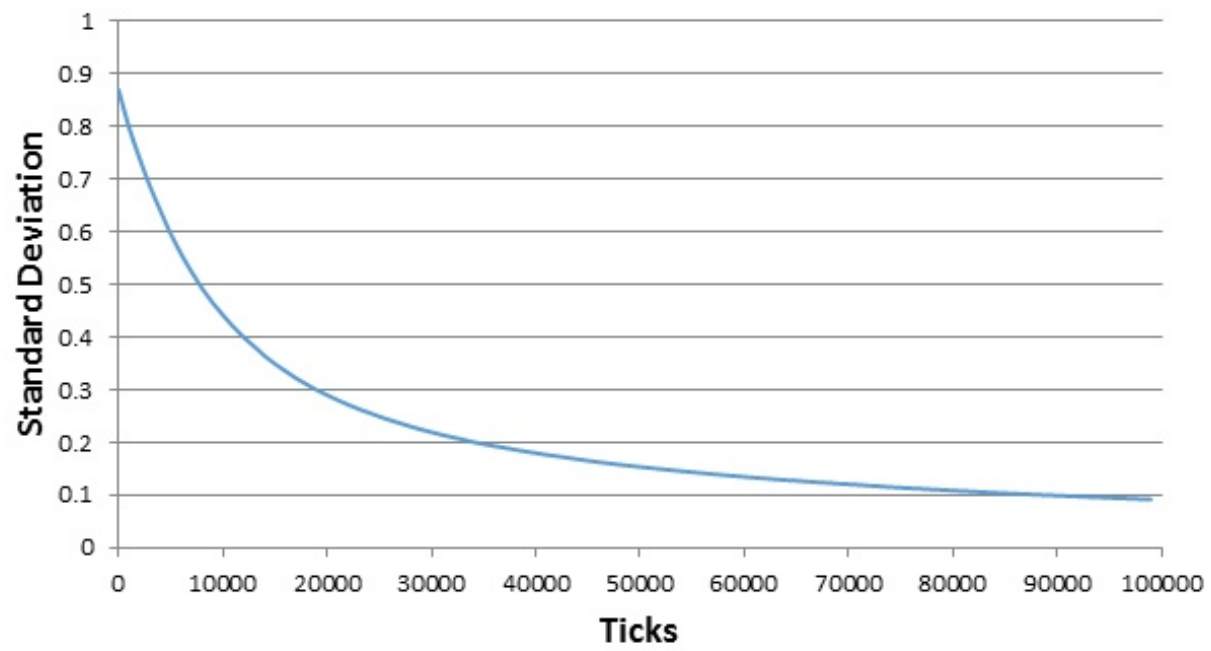

Figure 4: Decline in standard deviation in a histogram of agents' combined values on all features with word of mouth communication alone. Data sampled at intervals of 1000.

speed. The larger the meetings, and the more frequently they occur, the faster the population comes to a central consensus. Using standard deviation as a simple measure of distribution, Figure 6 shows the correlation 


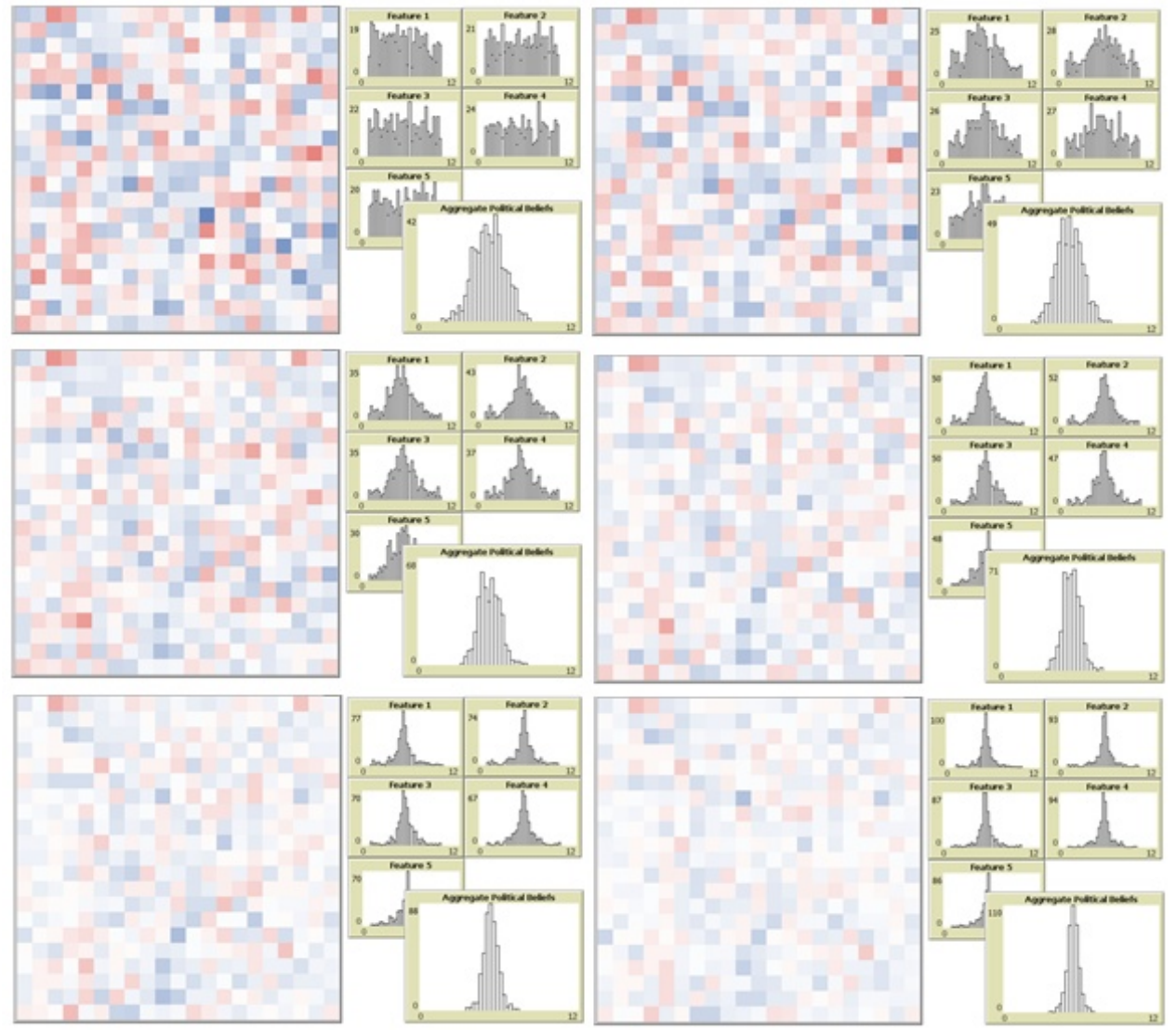

Figure 5: The convergence effect of town meetings. Medium-sized meetings, radius 7, at intervals of 25 ticks. Generations 0, 200, 400, 600, 800 and 1000 shown.

Standard deviation as a measure of convergence: Town meeting effects by size

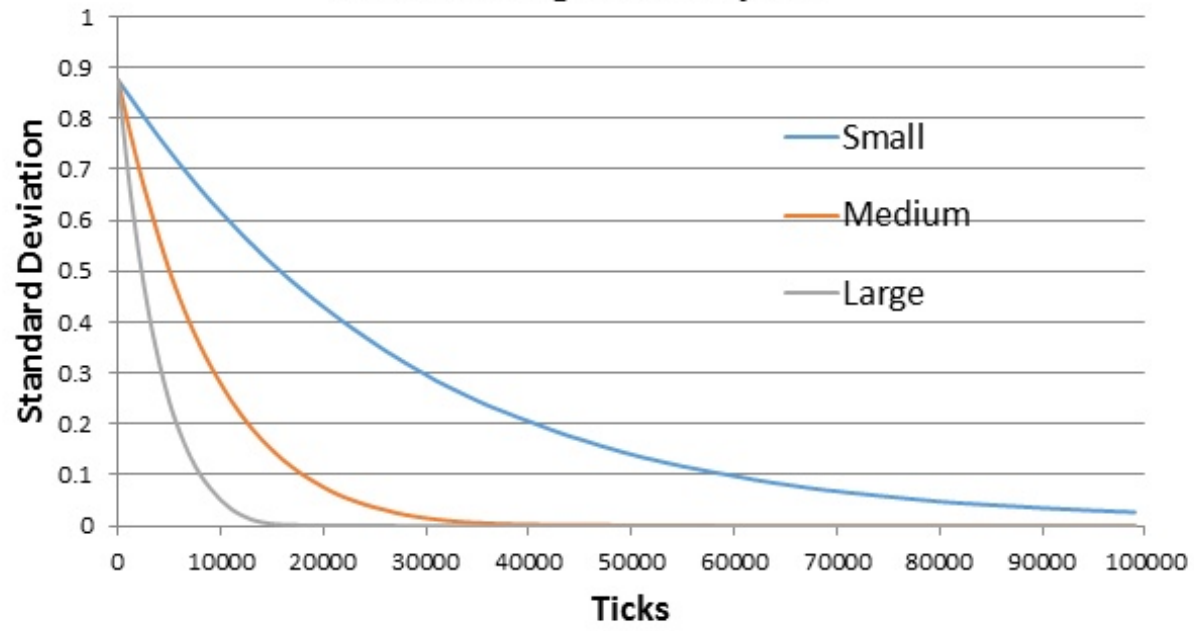

Figure 6: Standard deviation as a measure of speed of convergence for different meeting sizes. Meeting sizes of 49, 149, and 317 individuals. All meetings at intervals of 200 ticks.

between size of town meetings and speed to consensus.

3.8 To this point, using either word of mouth or town meetings, the lesson of the model is simply a lesson in opinion convergence. Both word of mouth and town meetings are forces toward agreement of opinion across a population ${ }^{6}$. Results turn out to be importantly different with self-selected media. 

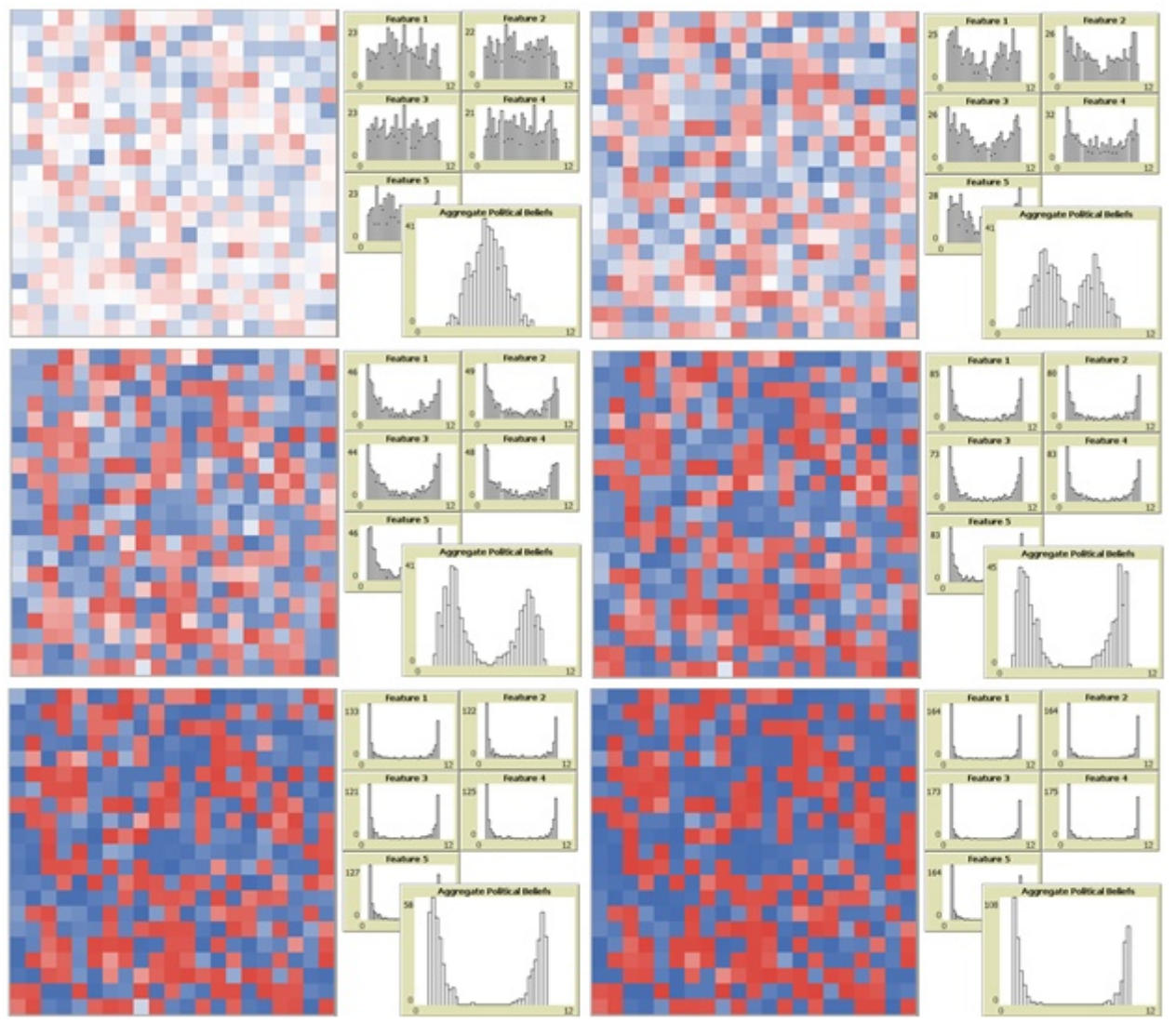

Figure 7: Popular polarization as an effect of self-selected media. Media at 1 and 10, with 10\% of the population interacting each 100 ticks with the closest media source with a probability correlate to similarity. Generations $0,50,100,150,200$ and 250 shown.

\section{Self-selected media}

3.9 Given self-selection of polarized media sources as outlined above, results show opinion bifurcation across the population, with each side drifting toward the closest media source (Figure 7$)^{7}$. Simulation results are in line with empirical evidence (Pew Research Center 2012; Prior 2013) and follow precisely the pattern background intuitions would suggest.

3.10 The reinforcement schedule for the possibility of media exposure, like for group meetings, can be set at various intervals. For every 100, 200, or 300 ticks at which individuals interact with neighbors, a certain percentage of the population - here set at an arbitrary $10 \%$ - is exposed to the similarity-based probability of interaction with the closest media source. Like word of mouth influence from immediate neighbors, probability of interaction is determined by similarity between opinion configurations in the individual and the closest media source 8 . Figure 8 shows standard deviation as a measure for polarization over time for different intervals of media exposure.

\section{Interaction of Media and Town Meetings}

3.11 The most interesting and complex results appear when we put both influences into play. Results for the interaction of the two social reinforcers acting together are shown in Figure 9. With media influence as outlined every 50 ticks and medium-sized town meetings at the same rate, standard deviation remains high: a sign of sustained polarized bifurcation at the ends of the spectrum. As media influence appears at longer intervals after only every 100 ticks, 150, or 200 - effects of convergence slowly overpower polarized bifurcation over the course of a run. With media accessed at 100 ticks or above, the dominant effect of town meetings results in a convergence of opinion across the community.

3.12 Hidden in standard deviation as a single measure, however, is an important interference effect between our two social reinforcers. What is particularly worthy of note is a dynamic involving two successive movements in the 


\section{Standard deviation as a measure of polarization: Effects of media intervals}

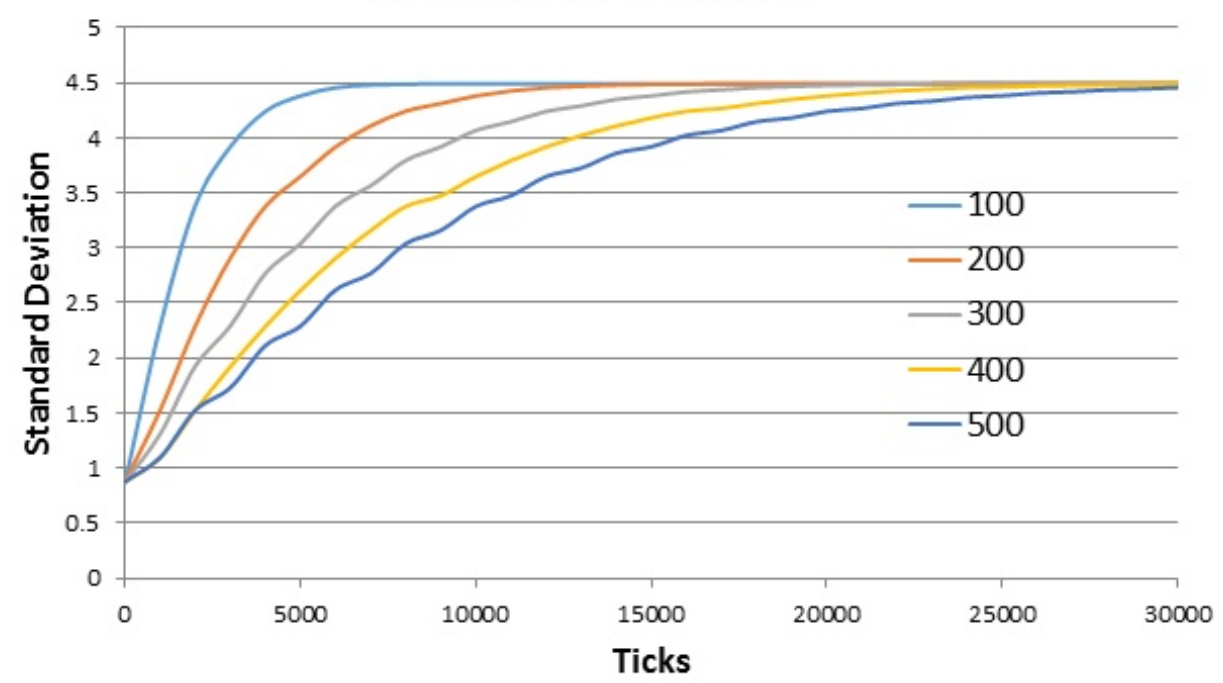

Figure 8: Increasing standard deviation as a measure of polarization for different media intervals. Media at 1 and 10 , all individuals interacting with most similar media source with probability correlate to similarity. Data sampled at intervals of 1000 ticks.

\section{Influence of media at various intervals with medium town meetings at intervals of 50}

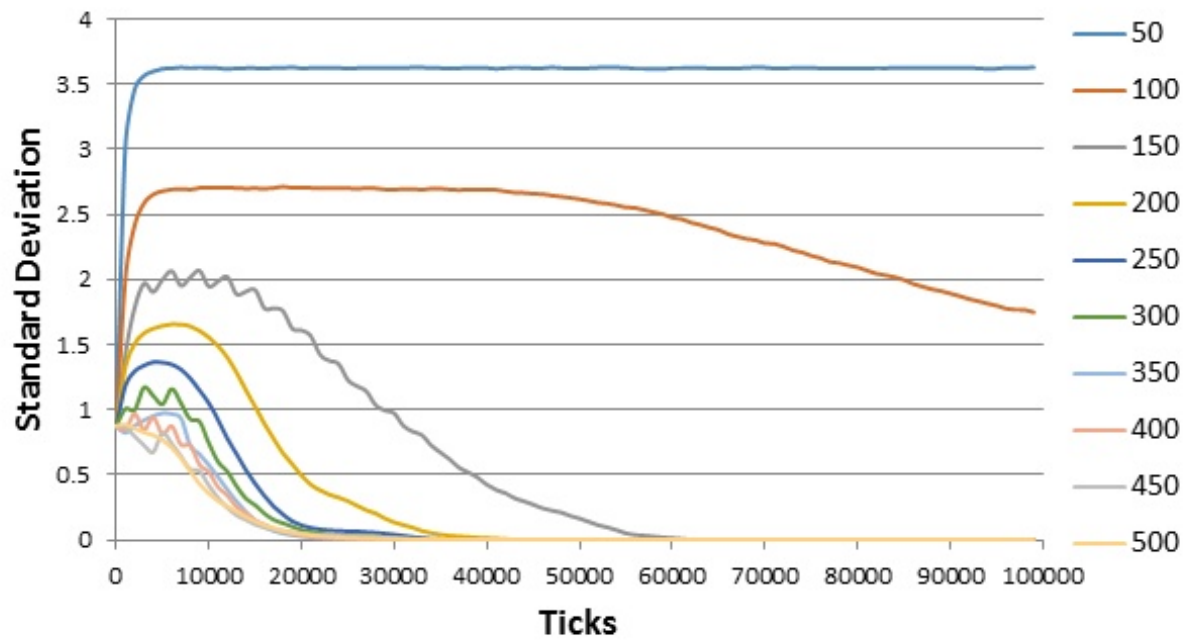

Figure 9: Standard deviation for medium meetings at intervals of 50 ticks with various rates of self-selected media influence. Note patterns of initial polarization followed by convergence. In all of the latter cases convergence is at one of the extreme ends of the opinion spectrum. Data sampled at each 1000 ticks.

pattern of development. Consider for example the development of standard deviation in Figure 9 at a media rate of 150,200 , or 250 . Here standard deviation first rises-indicating a polarized bifurcation in opinion-and only then converges ${ }^{9}$.

3.13 What is happening is illustrated in the successive frames and histograms of Figure 10. What first appears is a polarized bifurcation of opinion, familiar from the pattern of media influence. This is then followed by opinion convergence, on the pattern of town meetings. But with the media influence in place, the convergence that results is not at the center of the opinion spectrum but rather at one of the extremes defined by the media 


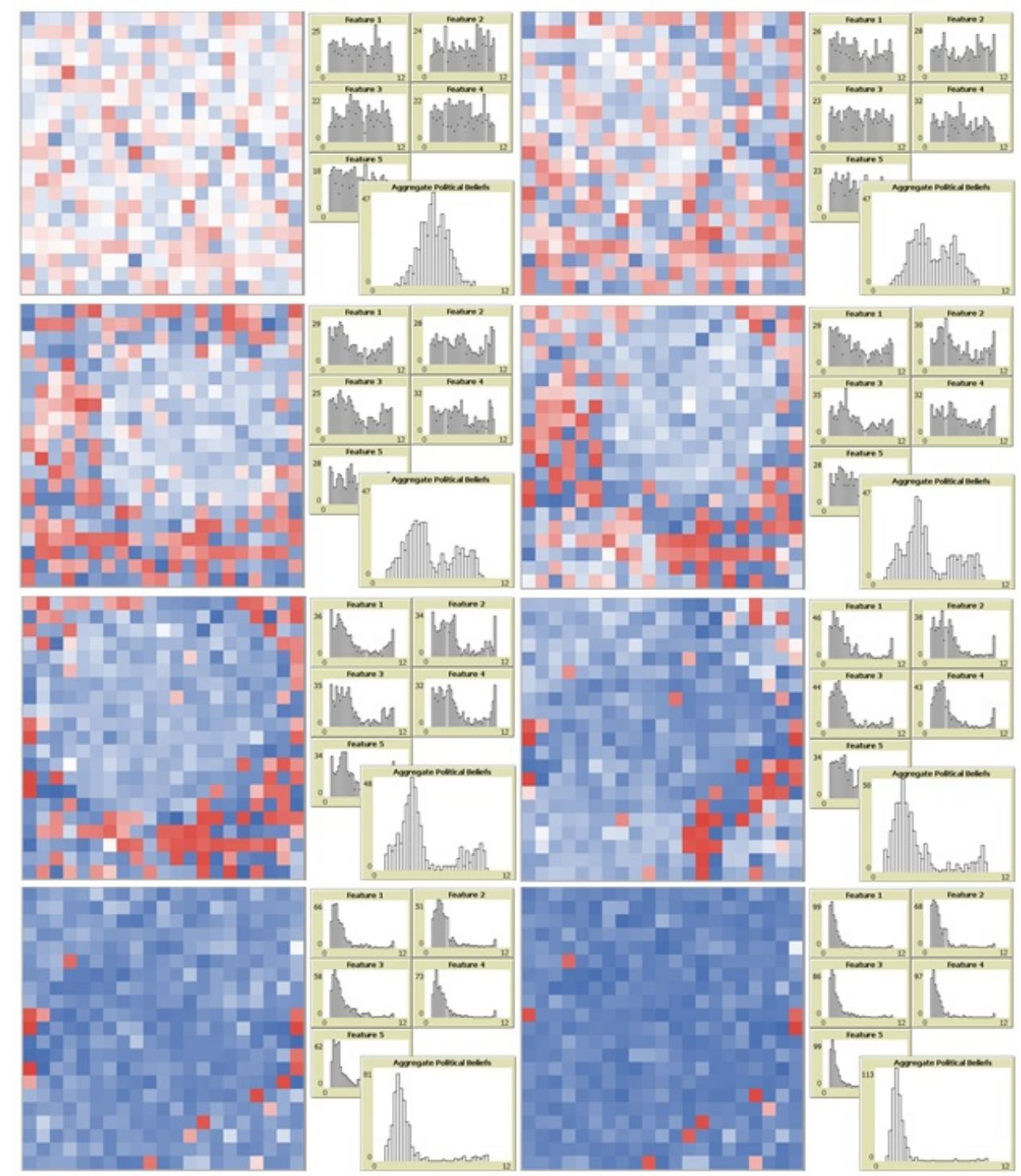

Figure 10: Interaction effect of medium-sized town meetings at intervals of 50 ticks with self-selected media influence at intervals of 300 ticks. Initial polarization is followed by convergence, but convergence at one of the extremes.

sources.

3.14 The influences of self-selected media and of town meetings are thus not merely competitive. It is not merely that one will dominate, with the result being either central convergence or bifurcated polarization. Where influence via town meetings is more frequent than that of self-selected media, holding constant meetings of medium size, the modelled social dynamics produces neither central convergence nor bifurcated polarization. The result is indeed convergence, as in the effect of town meetings alone, but convergence at one end of the spectrum of opinion-an effect clearly influenced by polarized media, but not one that is an effect of media alone.

\section{The Scope of Interaction Effects}

3.15 How pervasive is the interaction effect of media and town meetings observed above? In order to understand which parameter sets yield which outputs, we enlarge our array and re-tool the model to standardize the effects of media and town hall meeting interactions. In this form the variable for each influence is not the tick-interval of exposure but percentage of the population exposed to an influence at each tick of the simulation. At each tick, individuals in a $200 \times 200$ array interact with neighbors in terms of similarity as before. At each tick, however, $Y \%$ of the population is also involved in a clustered town hall meeting. At each tick, with probability based on 


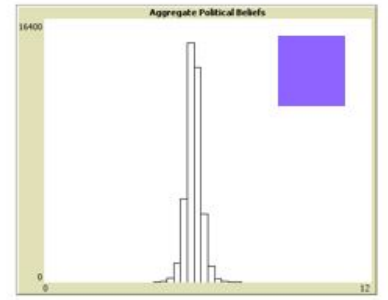

(a) Central global average, Low standard deviation

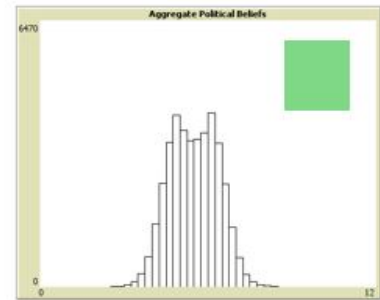

(b) Central global average, Medium standard deviation

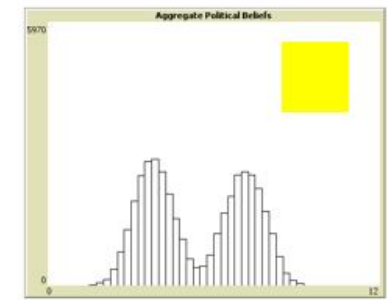

(c) Central global average, High standard deviation

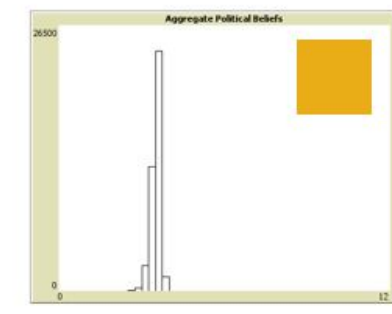

(d) Non-central global average, Medium standard deviation

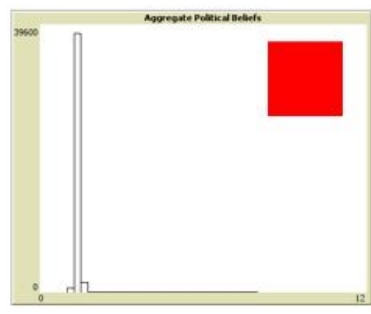

(e) Non-central global average, Low standard deviation

Figure 11: Color coding and sample histograms for various outcomes in the interaction of population selfselected media and town hall meetings. This color coding is used in Figure 12.

over-all similarity, $X \%$ of the population also interacts with their closest media source: our modeling for selfselected media exposure. By varying the percentage of the population involved at each tick in town meetings and self-selected media exposure we can explore interaction effects without some of the complexities tracked with temporal scheduling. Standard deviation was used above as a means of judging the end state of opinion across the population. Here we attempt a more complete view. Using measures for global average belief values and standard deviation together, we distinguish the range of possible outcomes shown in Figure 11.

3.16 The outcome (a) in which there is a close convergence of belief at the center of the political spectrum will have an approximately central value for global average belief and a very small standard deviation. That outcome will be coded in purple. An outcome (b) with central convergence that is somewhat less pronounced will have a central value for global average and a moderate standard deviation, coded in green. Outcome (c) in which there is polarized bifurcation on each side of the center will retain a central value for global average but have wide standard deviation, coded in yellow. The remaining two cases mark convergence at one end of the spectrum. Case (d), indicating moderate movement to one side in terms of a non-central average and moderate standard deviation, is indicated in a light brown. Case (e) is of particular interest: strong convergence on one extreme of the spectrum, indicated by non-central average and low standard deviation. This is the case we mark in red.

3.17 With color coding for these outcomes, we construct a heat map of interaction results using (i) percentages of the population involved in a town meeting each tick and (ii) percentages of the population which may be interacting with self-selected media each tick, each measured from 0 to $100 \%$ in steps of $1 \%$. The six images of Figure 12 show the belief distributions for 10,000 $200 \times 200$ arrays at six stages of development.

3.18 At 25 ticks of development, belief distribution shows polarized bifurcation (yellow) for most of the sampled arrays. Arrays characterized by a high enough percentage involved in town hall meetings, however, still show the central consensus indicated by green. The rough distribution of green and yellow across parameter combinations that is evident at 25 ticks remains as something of a background to further stages of development $-a$ background against which another pattern plays out.

3.19 Starting at 50 ticks in the evolution, increasing numbers of arrays show a pattern of non-centralized convergence. First brown (non-central consensus with medium standard deviation) and then red (non-central consensus with low standard deviation) invade the heat map from the top. Red represents consensus at an opinion extreme, which over time appears in arrays with progressively smaller percentages involved in town meetings and self-selected media. Consensus at an extreme appears first for arrays that previously showed a centralized consensus (green), indicating a movement of consensus from the center to the extreme. But the same extreme consensus also eventually appears for arrays that previously showed a bimodal polarization (yellow), indicating a movement from opinion bifurcation to consensus at one end of the opinion spectrum. 

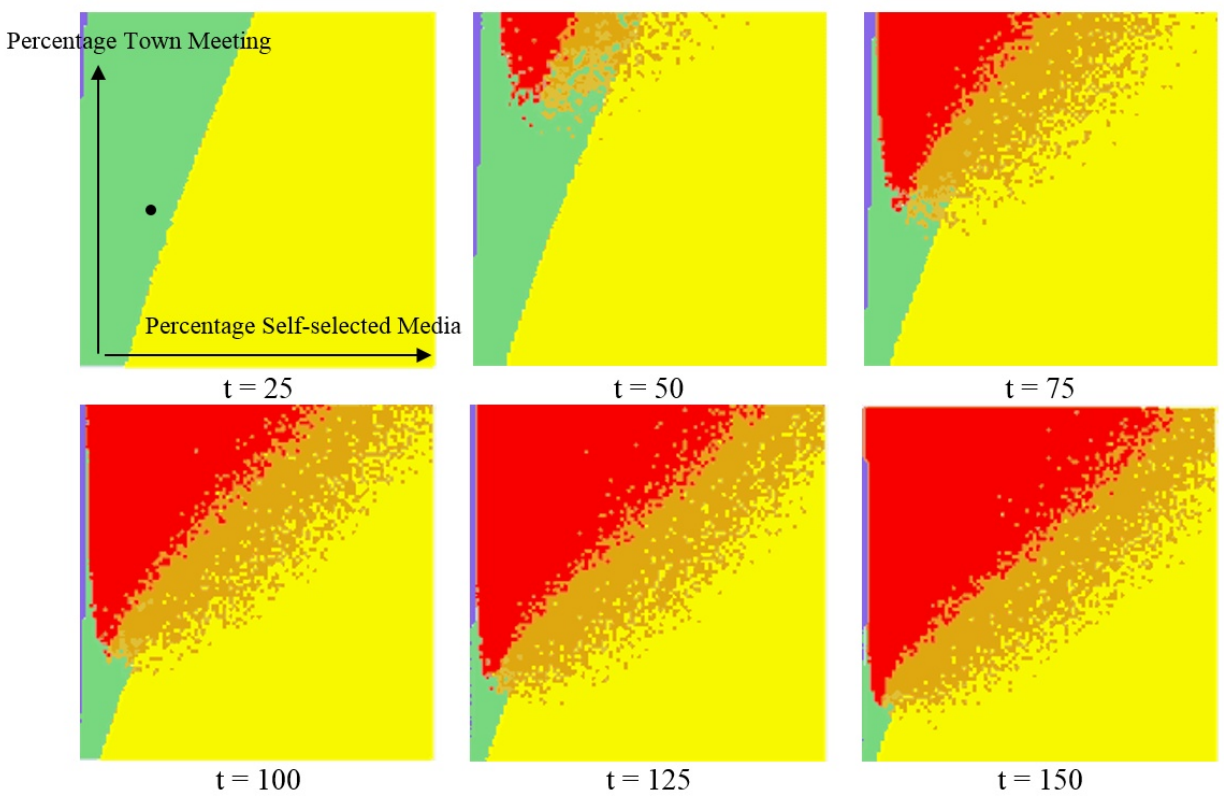

Figure 12: Outcomes of interaction effects developing over time, with t indicating ticks. Color coding is as in Figure 11, with purple and green for centralized consensus, yellow for polarized bifurcation and brown and red for convergence at one extreme of the belief spectrum.

3.20 Consider for example a single array from the 10,000 shown in each of the heat maps in Figure 12. At roughly the black spot in the first frame is an array in which $15 \%$ of the population is involved each tick in the self-selected media interaction outlined, with $40 \%$ of the population involved in a town-hall meeting. At 25 ticks, this array shows centralized consensus. By 100 ticks, it shows conversion to a non-central consensus (brown) to one side. By 150 ticks it shows a convergence at one extreme of the opinion scale (red). Indeed by 150 ticks the majority of arrays in the heat map, representing the majority of parameter combinations, show consensus at an extreme.

3.21 What the heat maps of parameter sweeps demonstrate is the robust pervasiveness of the effect outlined in the specific examples of earlier sections. Town hall meetings have a consensus effect: updating on the common wisdom of the group brings views together. Where media are themselves polarized, self-selected media show a polarizing effect on belief patterns. But where the two operate together, progressively across a wide range of parameter values for each, the result is neither polarized bifurcation nor central consensus. The effect of interaction between town meetings and self-selected media is opinion convergence at an extreme of the belief scale-an extreme that is itself set by one of the media sources in play. A number of model modifications remain for further exploration. Here, for example, percentages of the population involved in a town meeting are percentages included in a single town meeting; a percentage of $40 \%$ indicates a single town meeting in which $40 \%$ of the population participates. An alternative worth exploration is one in which the same proportion of the population is involved in some meeting or other, but in which that proportion may represent a sum of smaller meetings.

3.22 A number of model parameters have been set arbitrarily, including the amount that a numeric belief value changes as a result of an interaction. Variation of many such parameters remains to be explored, tied perhaps to experimental values from social psychology.

\section{Discussion}

4.1 Drawing on Axelrod's culture model (1997), with modifications in line with Deffuant et al. (2000, 2002, 2004), the topic of the current work is how interaction effects between two social reinforcers-town meetings and self-selected media-can shape public opinion. Simulation results follow background intuitions: town meetings produce a convergence of opinion at the center, while self-selected media polarize opinion at distinct media points. When the two reinforcers operate together, however, the result can be convergence at one of the extremes defined by media.

4.2 Viewed in terms of schedule, a clear example is the case of middle levels of meeting size (140 of 400 individuals) with meetings at intervals of 50 ticks and self-selected media influence tied to similarity at intervals of 150. Here 
opinion first shows polarized bifurcation, a clear effect of polarized media. At a later stage opinion converges, a clear influence of town meetings. Final convergence, however, is not central but at one extreme of the mediadefined poles of opinion (Figure 9).

4.3 The same effect appears clearly in a parameter sweep for percentages between 0 and 100 of the population involved in (i) town meetings and (ii) self-selected media in large $(200 \times 200)$ arrays. Results show a robust tendency developing over time for a majority of arrays to converge to consensus opinion at one of the mediadefined poles. The effect is clear and defined at 150 ticks for arrays in which town-meeting percentage equals or exceeds the percentage of individuals exposed to media influence with a probability geared to similarity (Figure 12).

4.4 One way of putting the result, phrased in terms of media influence, is this: Polarized media clearly have an effect on public opinion in the model, but have a very different effect depending on the presence or absence of town meetings. Without town meetings, polarized media result in polarized bifurcation of opinion. With town meetings, bifurcation is not a final result. Because of polarized media, however, the place at which consensus occurs is not central but toward an extreme. The results can also be phrased in terms of town meetings. Town meetings clearly have a consensus effect on public opinion in the model, but have a very different effect depending in the presence or absence of polarized media. Without polarized media, town meetings produce a central consensus. With polarized media they continue to have a consensus effect, but a consensus at one of the extremes defined by the media.

4.5 An intuitive mechanism can be seen to be driving these results. Individuals, initially with randomly assigned opinions, self-select toward different media sources. As individuals at different ends are pulled farther apart, the result is a distribution with wide standard deviation, either because it forms a broadened normal curve or an initial bimodal distribution. That wide distribution, however, will rarely be perfectly balanced. Given an unbalanced distribution, the convergence effect of town hall meetings will form a concentration of opinion at either one side of the center of the opinion spectrum or the other. Once the center of opinion is even slightly to one side or the other of the center, the pull of media on that side will continue, moving any established central consensus progressively toward one of the poles of media-defined opinion.

4.6 Ours is a modeling result, and as such is merely suggestive. What it suggests, however, is the intriguing hypothesis that the effects of (a) self-selected media and (b) group or town meeting deliberations should not be expected to function independently. There is clear psychological evidence that self-selected media polarize individual opinion in a community (lyengar \& Hahn 2009; Sunstein 2009; Stroud 2011; Pew Research Center 2012; Prior 2013). Common wisdom, at least, is that group discussion on the model of town hall meetings will promote convergence of opinion (Fishkin 1991, 1995, 2009; Fung 2006; Warren \& Pearse 2008; Niemeyer 2011; Grönlund et al. 2013). When the two social reinforcers are acting together, however, it should not be expected that they will simply balance each other out. Group or town meetings can be expected to have a consensus effect on opinion. Given constant enough influence from self-selection of polarized media, opinion bifurcation will be the rule. Where both forces are in play, the resulting pattern is often community consensus, but community consensus at one of the extremes defined by media. What our results suggest is that polarized media can shape the opinion landscape - 'define the question,' as it were - against which even convergence effects in group discussion, citizen forums, 'public spheres,' or 'mini-publics' will play out.

\section{Appendix: Pseudo-code}

Here is the model pseudocode.

\section{Notes}

${ }^{1}$ We have considered both Von Neumann and Moore neighborhoods, finding no important difference. We report Moore neighborhood results for word of mouth throughout.

${ }^{2}$ Intuitively, one might expect the entrenchment of opinion fragmentation to be even more severe with more than a few self-selected media sources, of a greater variety-websites, discussion groups, and blogs. Empirical evidence seems to suggest this is a more complex and nuanced question than might at first sight appear (Adamic \& Glance 2005; Hargittai et al. 2008; Gilbert et al. 2009). 
${ }^{3}$ McKeown \& Sheehy (2006) do not include town hall or interaction effects. It should be noted, however, that one of the regimes they describe is that we document here with a combination of media and town meetings: opinion convergence at one extreme after a period of persistent opinion exchange.

${ }^{4}$ We have found essentially the same effects starting with a normally distributed distribution of initial opinions.

${ }^{5}$ The crucial fact in both this model and others is that probabilities for interaction never reach absolute zero. That is the fundamental reason why Axelrod diversity disappears with the addition of noise in Klemm et al. (2003a). The fact that interaction probabilities can reach zero when bounded confidence levels are added also explains how diversity can reappear in Flache \& Macy (2006).

${ }^{6}$ We have noted above that our model tracks psychological work on meeting influence in terms of a group prototype (Turner 1985). We have also explored an alternative interaction rule for town meetings in which each agent has a single interaction with each of all other agents in the group, acting with more distant agents in the meeting as if they were immediate neighbors. Results for this alternative showed no significant difference.

${ }^{7}$ Convergence at each end of the polarization is on the position of the media sources. Here we have used extreme positions, giving convergence is at those extremes. In cases where we position media at .25 and .75, say, we get polarized convergence to those points.

${ }^{8}$ Experimentation with different population percentages shows only predictable results: exposing $20 \%$ of the population at intervals of 100 ticks gives results essentially identical to exposing $10 \%$ at intervals of 200 .

${ }^{9}$ The visible oscillation at an interval of 350 is due to the fact that our sampling is at 1000 ticks. At an interval of 350 , one of our forces (town meetings or self-selected meetings) may be dominant at one sampling point, with the other dominant at the next.

\section{References}

Adamic, L. A. \& Glance, N. (2005). The political blogosphere and the 2004 US election: Divided they blog. In Proceedings of the 3rd International Workshop on Link Discovery, (pp. 36-43). doi:10.1145/1134271.1134277

Axelrod, R. (1997). The dissemination of culture: A model with local convergence and global polarization. Journal of Conflict Resolution, 41(2), 203-226

Bennett, W. L. \& Iyengar, S. (2008). A new era of minimal effects? the changing foundations of political communication. Journal of Communication, 58(4), 707-731. doi:10.1111/j.1460-2466.2008.00410.x

Bishop, B. (2008). The Big Sort: Why the Clustering of Like-minded America Is Tearing Us Apart. Boston, MA: Houghton Mifflin

Brechner, K. C. (1977). An experimental analysis of social traps. Journal of Experimental Social Psychology, 13(6), 552-564

Brechner, K. C. \& Linder, D. (1981). A social trap analysis of energy distribution systems. Advances in Environmental Psychology, 3, 27-51

Bryan, F. M. (2004). Real Democracy: The New England Town Meeting and How It Works. Chicago, IL: University of Chicago Press

Castellano, C., Marsili, M. \& Vespignani, A. (2000). Nonequilibrium phase transition in a model for social influence. Physical Review Letters, 85(16), 3536

Deffuant, G., Amblard, F. \& Weisbuch, G. (2004). Modelling group opinion shift to extreme: The smooth bounded confidence model. arXiv preprint cond-mat/0410199

Deffuant, G., Amblard, F., Weisbuch, G. \& Faure, T. (2002). How can extremism prevail? a study based on the relative agreement interaction model. Journal of Artificial Societies and Social Simulation, 5(4), 1

Deffuant, G., Neau, D., Amblard, F. \& Weisbuch, G. (2000). Mixing beliefs among interacting agents. Advances in Complex Systems, 3(01n04), 87-98. doi:10.1142/S0219525900000078

Dittmer, J. C. (2001). Consensus formation under bounded confidence. Nonlinear Analysis: Theory, Methods \& Applications, 47(7), 4615-4621 
Ferster, C. B. \& Skinner, B. F. (1957). Schedules of Reinforcement. East Norwalk, CT: Appleton-Century-Crofts. doi:10.1037/10627-000

Festinger, L. (1954). A theory of social comparison processes. Human Relations, 7(2), 117-140

Fiorina, M. P. \& Levendusky, M. S. (2006). Disconnected: The political class versus the people. In R. S. Nivola \& D. W. Brady (Eds.), Red and Blue Nation: Characteristics, Causes, and Consequences of America's Polarized Politics, (pp. 49-71). Washington, DC: Brookings Institution Press

Fishkin, J. S. (1991). Democracy and Deliberation: New Directions for Democratic Reform. New Haven: Yale University Press

Fishkin, J. S. (1995). The Voice of the People: Public Opinion and Democracy. New Haven: Yale University Press

Fishkin, J. S. (2009). When the People Speak: Deliberative Democracy and Public Consultation. Oxford: Oxford University Press

Flache, A. \& Macy, M. W. (2006). What sustains cultural diversity and what undermines it? axelrod and beyond. ArXiv Physics e-prints

Fung, A. (2006). Democratizing the policy process. In R. Goodin, M. Moran \& M. Rien (Eds.), Oxford Handbook of Public Policy, (pp. 669-685). New York: Oxford University Press

Galam, S. (1997). Rational group decision making: A random field Ising model at T $=0$. Physica A: Statistical Mechanics and its Applications, 238(1), 66-80. doi:10.1016/S0378-4371(96)00456-6

Gilbert, E., Bergstrom, T. \& Karahalios, K. (2009). Blogs are echo chambers: Blogs are echo chambers. In System Sciences, 2009. HICSS '09. 42nd Hawaii International Conference on, (pp. 1-10). doi:10.1109/HICSS.2009.91

González-Avella, J. C., Cosenza, M. G., Klemm, K., Eguíluz, V. M. \& Miguel, M. S. (2007). Information feedback and mass media effects in cultural dynamics. arXiv preprint arXiv:0705.1091

Grönlund, K., Bächtiger, A. \& Setälä, M. (Eds.) (2013). Deliberative Mini-Publics: Practices, Promises, Pitfalls. New York, NY: Columbia University Press

Habermas, J. (1996). Between Facts and Norms: Contributions to a Discourse Theory of Law and Democracy. Cambridge, MA: MIT Press

Hargittai, E., Gallo, J. \& Kane, M. (2008). Cross-ideological discussions among conservative and liberal bloggers. Public Choice, 134(1-2), 67-86

Hegselmann, R., Krause, U. et al. (2002). Opinion dynamics and bounded confidence models, analysis, and simulation. Journal of Artificial Societies and Social Simulation, 5(3), 2

Iyengar, S. \& Hahn, K. S. (2009). Red media, blue media: Evidence of ideological selectivity in media use. Journal of Communication, 59(1), 19-39. doi:10.1111/j.1460-2466.2008.01402.x

Kacperski, K. \& Hołyst, J. A. (2000). Phase transitions as a persistent feature of groups with leaders in models of opinion formation. Physica A: Statistical Mechanics and its Applications, 287(3), 631-643. doi:10.1016/ S0378-4371(00)00398-8

Klemm, K., Eguíluz, V. M., Toral, R. \& Miguel, M. S. (2003a). Global culture: A noise-induced transition in finite systems. Physical Review E, 67, 045101. doi:10.1103/PhysRevE.67.045101

Klemm, K., Eguíluz, V. M., Toral, R. \& San Miguel, M. (2003b). Role of dimensionality in axelrod's model for the dissemination of culture. Physica A: Statistical Mechanics and its Applications, 327(1-2), 1 - 5. doi:10.1016/ S0378-4371(03)00428-X

Leeper, T. J. (2014). The informational basis for mass polarization. Public Opinion Quarterly, 78(1), 27-46. doi: $10.1093 / \mathrm{poq} / \mathrm{nft} 045$

Levendusky, M. (2013). How Partisan Media Polarize America. Chicago, IL: University of Chicago Press

Levendusky, M. S. (2014). Why do partisan media polarize viewers? American Journal of Political Science, 57(3), 611-623. doi:10.1111/ajps.12008 
Madden, G. J. (2013). APA Handbook of Behavioral Analysis. Washington, DC: American Psychological Association

Mason, W. A., Conrey, F. R. \& Smith, E. R. (2007). Situating social influence processes: Dynamic, multidirectional flows of influence within social networks. Personality and Social Psychology Review, 11(3), 279-300. doi: $10.1177 / 1088868307301032$

McKeown, G. \& Sheehy, N. (2006). Mass media and polarisation processes in the bounded confidence model of opinion dynamics. Journal of Artificial Societies and Social Simulation, 9(1), 11

McPherson, M., Smith-Lovin, L. \& Cook, J. M. (2001). Birds of a feather: Homophily in social networks. Annual Review of Sociology, 27, 415-444. doi:doi:10.1146/annurev.soc.27.1.415

Morgan, D. L. (2010). Schedules of reinforcement at 50: A retrospective appreciation. The Psychological Record, 60(1), 151-172

Niemeyer, S. (2011). The emancipatory effect of deliberation: Empirical lessons from mini-publics. Politics \& Society, 39(1), 103-140. doi:10.1177/0032329210395000

Pew Research Center (2012). News consumption survey. Http://www.people-ress.org/files/legacy-pdf/2012

Prior, M. (2013). Media and political polarization. Annual Review of Political Science, 16(1), 101-127. doi:10.1146/ annurev-polisci-100711-135242

Shibanai, Y., Yasuno, S. \& Ishiguro, I. (2001). Effects of global information feedback on diversity extensions to Axelrod's adaptive culture model. Journal of Conflict Resolution, 45(1), 80-96. doi:10.1177/ 0022002701045001004

Skinner, B. F. (1981). Selection by consequences. Science, 213(4507), 501-504

Skocpol, T. (2013). Diminished Democracy: From Membership to Management in American Civic Life. Norman, OK: University of Oklahoma press

Stroud, N. J. (2011). Niche News: The Politics of News Choice. New York, NY: Oxford University Press

Sunstein, C. R. (2009). Going to Extremes: How Like Minds Unite and Divide. New York, NY: Oxford University Press

Sustein, C. R. (2002). Republic.com. Princeton, NJ: Princeton University Press

Turner, J. C. (1985). Social categorization and the self-concept: A social cognitive theory of group behavior. Advances in Group Processes, 2, 77-122

Warren, M. E. (2009). Two trust-based uses of mini-publics in democracy. In Proceedings of the 2009 American Political Science Association Meeting. Toronto

Warren, M. E. \& Pearse, H. (2008). Designing Deliberative Democracy: The British Columbia Citizens' Assembly. Cambridge, MA: Cambridge University Press. doi:10.1017/CBO9780511491177

Wilkie, D. M. (1972). Variable-time reinforcement in multiple and concurrent schedules. Journal of the Experimental Analysis of Behavior, 17(1), 59-66. doi:10.1901/jeab.1972.17-59

Williams, B. A. (1983). Another look at contrast in multiple schedules. Journal of the Experimental Analysis of Behavior, 39(2), 345-384. doi:10.1901/jeab.1983.39-345

Williams, B. A. (1994). Conditioned reinforcement: Neglected or outmoded explanatory construct? Psychonomic Bulletin \& Review, 1(4), 457-475. doi:10.3758/BF03210950

Zeiler, M. (1977). Schedules of reinforcement: The controlling variables. In W. Honig \& J. Staddon (Eds.), Handbook of Operant Behavior, (pp. 201-232). Englewood Cliffs: Prentice Hall 